Article

\title{
Facile One-Step Microwave-Assisted Route towards Ni Nanospheres/Reduced Graphene Oxide Hybrids for Non-Enzymatic Glucose Sensing
}

\author{
Zhigang Wang ${ }^{1}$, Yong Hu ${ }^{1, *}$, Wenlong Yang ${ }^{1}$, Mojiao Zhou ${ }^{1}$ and Xiao Hu ${ }^{2, *}$ \\ 1 Institute of Physical Chemistry, Zhejiang Normal University, Jinhua 321004, China; \\ E-Mails: zhigangwang@zjnu.net (Z.W.); yangwenlong@zjnu.net (W.Y.); \\ zhoumojiao@zjnu.net (M.Z.)
}

2 School of Materials Science \& Engineering, Nanyang Technological University, 639798, Singapore

* Authors to whom correspondence should be addressed; E-Mails: yonghu@zjnu.edu.cn (Y.H.); asxhu@ntu.edu.sg (X.H.); Tel.: +86-579-8228-2234; Fax: +86-579-8228-2595.

Received: 24 February 2012; in revised form: 14 March 2012 / Accepted: 23 March 2012 /

Published: 13 April 2012

\begin{abstract}
In this work, a facile one-step microwave-assisted method for deposition of monodisperse $\mathrm{Ni}$ nanospheres on reduced graphene oxide (rGO) sheets to form Ni-rGO nanohybrids is discussed. In the presence of hydrazine monohydrate, Ni nanospheres are grown onto rGO sheets using nickel precursor and GO as starting materials in ethylene glycol (EG) solution under a low level of microwave irradiation $(300 \mathrm{~W})$ for $20 \mathrm{~min}$, during which $\mathrm{GO}$ is also reduced to rGO. The as-prepared nanohybrids exhibit well-dispersed Ni nanosphere (about $80 \mathrm{~nm}$ in diameter) loadings and effective reduction of graphene oxide. The resulting Ni-rGO nanohybrids-modified glassy carbon electrode (GCE) shows significantly improved electrochemical performance in nonenzymatic amperometric glucose detection. In addition, interference from the oxidation of common interfering species under physiological conditions, such as ascorbic acid (AA) and uric acid (UA), is effectively avoided.
\end{abstract}

Keywords: carbon materials; nanocomposites; microwave-assisted method; sensors 


\section{Introduction}

The development of fast and reliable methods for glucose detection is of considerable importance due to its extremely important applications in clinical diagnosis, food analysis, and bioreactor monitoring [1,2]. Up to now, the glucose oxidase (GOD)-based sensors with high selectivity have been widely studied [3-5], but GOD-based biosensors suffer from a stability problem as the enzyme can be easily affected by temperature, $\mathrm{pH}$ value, humidity and toxic chemicals [6]. To overcome these obstacles, there is great interest in the fabrication of nonenzymatic glucose sensors based on the direct oxidation of glucose for extended usage. Compared with GOD-based biosensors, the nonenzymatic glucose sensors possess higher sensibility, lower detection limits, less susceptibility to environmental factors, better stability and shorter response times [7-9].

Recently, Ni-based materials have been extensively investigated for electrocatalytic oxidation of glucose, since they could allow production of glucose sensors in large numbers at low cost [10-14]. The oxidation processes are catalyzed by the Ni-based materials through the formation of a high-valent, oxyhydroxide species [NiOOH] in alkaline medium [11]. Graphene, a single layer of carbon atoms tightly packed into a two-dimensional [2D] honeycomb $\mathrm{sp}^{2}$ carbon lattice, has a unique ability to promote fast electron transfer kinetics for a wide range of electroactive species $[15,16]$. Deposition of metal nanoparticles on graphene sheets gives rise to nanocomposites with larger active surface areas and enhanced electron transport, making the nanocomposites ideal materials for the fabrication of electrochemical sensing devices [17]. It has been reported that graphene may play a distinct role in improving the conductivity of Ni-based materials for glucose sensors, in which the electron transfer is quick, effectively enhancing the detecting sensitivity and shortening the response times [18-20]. Very recently, chemical vapor deposition has increased in popularity in the synthesis of graphene/Ni nanohybrids, although the processes are complicated and hence difficult to scale [21,22].

Microwave irradiation is an attractive and facile method for the rapid synthesis of nanocrystals with small particle size, narrow particle size distribution, and high purity. Compared with conventional heating, it has a more homogeneous heating process. Moreover, it can promote nucleation and reduce the synthesis times considerably, generating smaller and more uniform particles [23-25]. Herein, we demonstrated a facile one-step microwave-assisted method to directly deposit Ni nanospheres on reduced graphene oxide (rGO) sheets (Scheme 1). When a GO sheet solution is mixed with a nickel salt solution, $\mathrm{Ni}^{2+}$ is selectively bonded with carboxyl through mutual electrostatic attraction. Under continuous stirring conditions, the interlayer spacing gradually increases and $\mathrm{Ni}^{2+}$ could interlaminate more easily into the enlarged layer [26]. In the presence of hydrazine monohydrate, Ni nanospheres were grown onto rGO sheets in ethylene glycol (EG) solution under a low level of microwave irradiation $(300 \mathrm{~W})$ for $20 \mathrm{~min}$, during which GOs were also reduced to rGO. These nanocomposites exhibit well-dispersed $\mathrm{Ni}$ nanosphere (about $80 \mathrm{~nm}$ in diameter) loadings and effective reduction of graphene oxide. By forming the rGO-supported Ni nanospheres (Ni-rGO nanohybrids), one might be able to take advantage of the best features of both components. As expected, the as-prepared Ni-rGO nanohybrid-modified glassy carbon electrode (GCE) shows highly sensitivity and fast amperometric sensing of glucose. In addition, interference from the oxidation of common interfering species present in body fluids, such as ascorbic acid (AA) and uric acid (UA), is effectively avoided. 
Scheme 1. Schematic illustration of the formation of Ni-rGO nanohybrids via a facile one-step solvothermal route at low temperature.

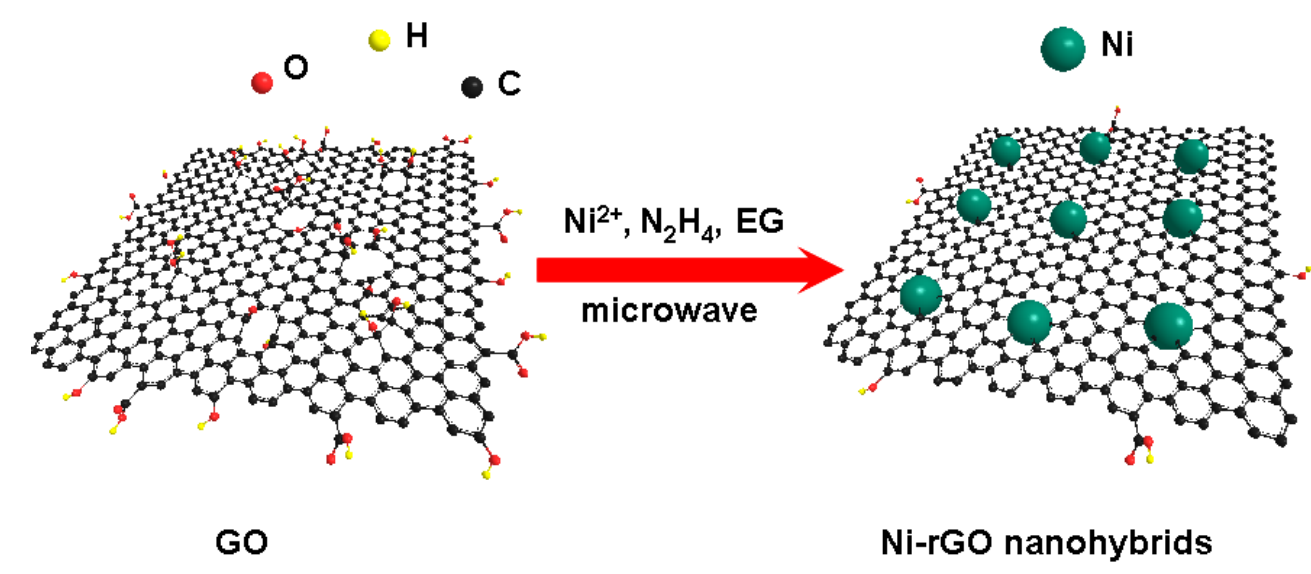

\section{Experimental Details}

All reagents were of analytical grade, purchased from the Shanghai Chemical Reagent Manufacturing Co., and used as received without further purification.

\subsection{Preparation of $G O$}

In a typical procedure, GO was first prepared from pure graphite using a modified Hummer's method [27,28]. Briefly, $2.0 \mathrm{~g}$ of natural graphite powder was added to $300 \mathrm{~mL}$ of $\mathrm{H}_{2} \mathrm{SO}_{4}$ under stirring at $0{ }^{\circ} \mathrm{C}$, and then $3.0 \mathrm{~g}$ of $\mathrm{NaNO}_{3}$ and $20 \mathrm{~g}$ of $\mathrm{KMnO}_{4}$ were added gradually. Successively, the mixture was transferred to a water bath at $30^{\circ} \mathrm{C}$ and stirred for $20 \mathrm{~min}$ to form a thick paste. Then, $250 \mathrm{~mL}$ of distilled water was slowly added and the temperature was increased to $98{ }^{\circ} \mathrm{C}$. After 30 min aging, another $500 \mathrm{~mL}$ of water was added and this was followed by a dropwise addition of $40 \mathrm{~mL}$ of $\mathrm{H}_{2} \mathrm{O}_{2}$ $(30 \%)$. When the color of the solution changed from dark brown to brilliant yellow, the mixture was filtered and washed with diluted $\mathrm{HCl}$ aqueous $(1 / 10 \mathrm{v} / \mathrm{v})$ three times to remove metal ions, and then washed with distilled water repeatedly until the $\mathrm{pH}$ became 7 . Finally, the as-prepared GO was obtained after drying in a vacuum oven at room temperature.

\subsection{One-Step Microwave-Assisted Growth of Ni Nanospheres on rGO Sheets}

Next, $0.058 \mathrm{~g}$ of $\mathrm{Ni}\left(\mathrm{NO}_{3}\right)_{2} \cdot 6 \mathrm{H}_{2} \mathrm{O}$ and $10 \mathrm{mg}$ of the as-prepared $\mathrm{GO}$ were dissolved in $40 \mathrm{~mL}$ of EG with the assistance of ultrasonication for $1 \mathrm{~h}$. Subsequently, the mixture was transferred to an oil bath under vigorous stirring, and then $2 \mathrm{~mL}$ of hydrazine monohydrate $\left(\mathrm{N}_{2} \mathrm{H}_{4} \cdot \mathrm{H}_{2} \mathrm{O}, 85 \%\right)$ was slowly added and the solution was placed in a microwave refluxing system irradiated at $300 \mathrm{~W}$ for $20 \mathrm{~min}$. Finally, the as-prepared products were collected and thoroughly rinsed several times with distilled water and ethanol and then dried at $60{ }^{\circ} \mathrm{C}$ for $12 \mathrm{~h}$.

\subsection{Characterization}

Powder X-ray diffraction (XRD) measurements of the samples were performed with a Philips PW3040/60 X-ray diffractometer using $\mathrm{Cu}_{\mathrm{K} \alpha}$ radiation at a scanning rate of $0.06^{\circ} \mathrm{s}^{-1}$. Fourier transform 
infrared (FT-IR) spectra were recorded on a Nicolet NEXUS670 FT-IR spectrometer using KBr pellets. Scanning electron microscopy (SEM) was performed with a Hitachi S-4800 scanning electron microanalyzer with an accelerating voltage of $15 \mathrm{kV}$. Transmission electron microscopy (TEM) and high resolution TEM were conducted at $200 \mathrm{kV}$ with a JEM-2100F field emission TEM, after dispersing the Ni-rGO nanohybrids in ethanol and depositing several drops of the suspension on the carbon coated copper grids and dried under ambient conditions.

\subsection{Electrochemical Measurements}

All electrochemical measurements were conducted using a CHI840C electrochemical workstation with conventional three-electrode setup at room temperature. A Ni-rGO hybrids modified GCEs was employed as working electrode, a saturated $\mathrm{Hg} / \mathrm{Hg}_{2} \mathrm{Cl}_{2}$ electrode (SCE) as reference electrode and platinum wire as counter electrode. The supporting electrolyte is $0.1 \mathrm{M} \mathrm{NaOH}$ containing $0.1 \mathrm{M} \mathrm{KCl}$, and deionized water was used throughout the experiments. For calibration experiment, amperometric measurements were carried out at $0.5 \mathrm{~V}$, while $100 \mu \mathrm{L}$ of 0.2 or $2 \mathrm{mM}$ glucose was added into $20 \mathrm{~mL}$ of electrolyte under a magnetically stirred condition to obtain a stepwise increase to reach a final concentration $1 \mu \mathrm{M}$ or $10 \mu \mathrm{M}$.

\section{Results and Discussion}

The XRD patterns of the as-prepared GO and Ni-rGO nanohybrids are shown in Figure 1. For GO, the characteristic peak at around $11.3^{\circ}$ (the d-spacing is about $0.79 \mathrm{~nm}$ ) is consistent with the interlayer spacing of GO sheets reported previously, which may be ascribed to the existence of oxygen-rich groups on both sides of the sheets and water molecules trapped between the sheets [29].

Figure 1. XRD patterns of the as-prepared GO and Ni-rGO nanohybrids.

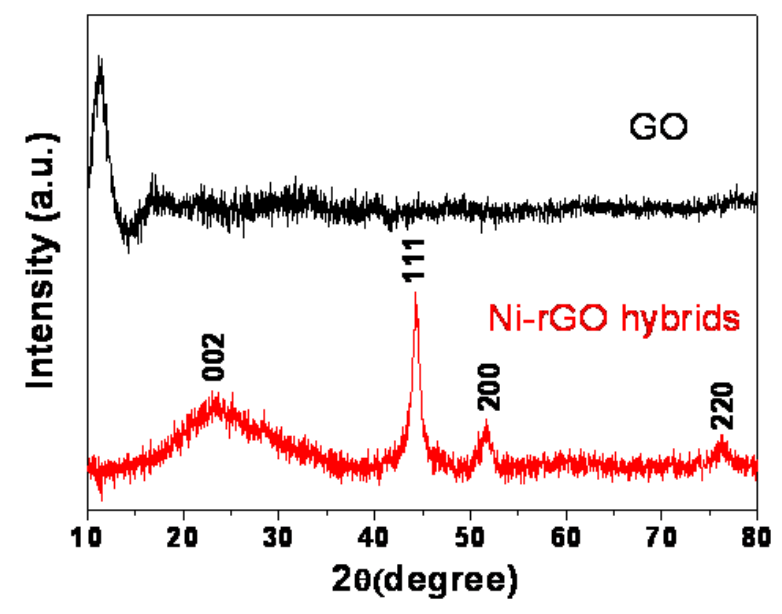

In the XRD pattern of the Ni-rGO nanohybrids, the clear diffraction bands centered at $2 \theta$ of $44.4^{\circ}$, $51.8^{\circ}$, and $76.3^{\circ}$ are corresponding to the (111), (200), and (220) crystal planes respectively of the face-centered cubic (fcc) Ni (JCPDS 04-0850, a $=0.3523 \mathrm{~nm}$ ). A broad hump at $23.2^{\circ}$ can be attributed to the (002) diffraction of rGO, indicating the successfully reduction of GO by hydrazine hydrate [30]. In addition, the average size of Ni nanoparticles is $9.4 \mathrm{~nm}$, which calculated using Debye-Scherrer equation based on the full width at half-maximum of the diffraction peak. 
Figure 2 shows the FT-IR spectra of the as-prepared of GO and Ni-rGO nanohybrids. The broad absorptions at about 3,425 and $1,625 \mathrm{~cm}^{-1}$ are assigned to the hydroxyl groups of absorbed $\mathrm{H}_{2} \mathrm{O}$ molecules, and the peaks around 2,970 and 2,900 $\mathrm{cm}^{-1}$ can be assigned to the asymmetric and symmetric vibrations of $\mathrm{C}-\mathrm{H}$, respectively. The absorption band at $1,087 \mathrm{~cm}^{-1}$ can be assigned to the stretching vibration of $\mathrm{C}-\mathrm{O}$. The $\mathrm{C}=\mathrm{O}$ vibration band at $1,723 \mathrm{~cm}^{-1}$ disappears after hydrazine hydrate reduction of exfoliated $\mathrm{GO}$, indicating that the reduction of $\mathrm{GO}$ to $\mathrm{rGO}$ is complete [31].

Figure 2. FT-IR spectra of the as-prepared GO and Ni-rGO nanohybrids.

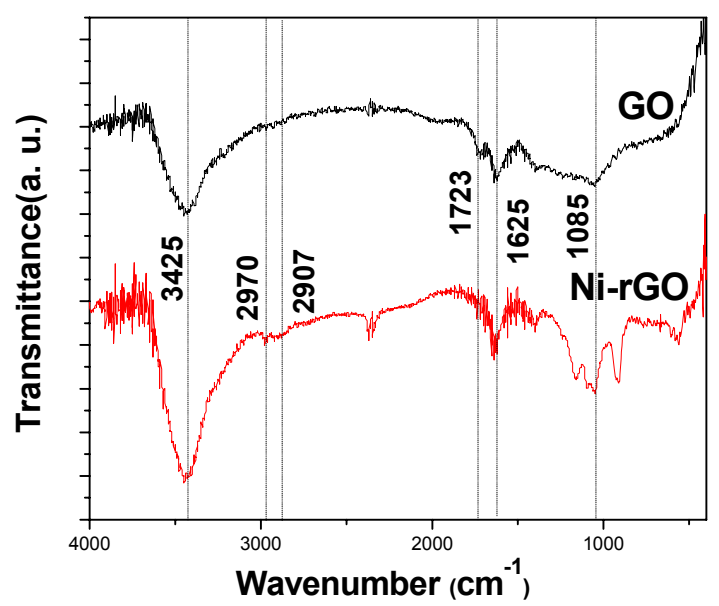

The SEM images reveal the morphology of the Ni-rGO nanohybrids prepared by our one-step microwave-assisted method. The low magnification SEM image (Figure 3(a)), reveals that the GO sheets are well exfoliated and nearly monodispersed $\mathrm{Ni}$ nanospheres are anchored uniformly on the surface of the rGO sheets. Observation under higher magnification (Figure 3(b)) shows the well-dispersed Ni spheres with a diameter of about $80 \mathrm{~nm}$ on the wrinkly rGO sheets. The transmission electron microcopy (TEM) images (Figure 3(c,d)) also show that these monodiperse spheres are about $80 \mathrm{~nm}$ in size, while the HRTEM image (inset in Figure 3(c)) indicates that there are smaller nanocrystals attached uniformly to form nanospheres. In our study, hydrazine does not only acts as a reducing agent to form $\mathrm{Ni}$ nanospheres in the subsequent solvothermal process, but also plays an important role in reducing GO to rGO sheets. GO, bearing hydroxyl and epoxide groups on the basal planes, along with carbonyl and carboxyl groups along the sheet edges, readily coordinates with $\mathrm{Ni}^{2+}$ from solution. The strong reducing ability of hydrazine hydrate ensures synchronous reduction of GO and nickel ions which leads to the formation of nanohybrids [32]. Additionally, we believe the EG solvent selected for this experiment also acts as a surfactant [10], which absorbs on the GO surface, while at the same time providing hydrophilic hydroxyl groups for the hydrolysis of the nickel precursor. Hence, GO can be used as a template for deposition of Ni nanospheres on its surface.

To investigate the electrocatalytic activity of the as-prepared Ni-rGO hybrid-modified GCE toward the oxidation of glucose, the corresponding cyclic voltammograms (CVs) were obtained in the absence and presence of glucose in $0.1 \mathrm{M}$ of $\mathrm{NaOH}$ solution at a potential sweep rate of $0.1 \mathrm{Vs}^{-1}$. Figure 4 shows the CVs of the as-prepared GO and Ni-rGO hybrid-modified GCE in the 0.1 M NaOH solution in the absence glucose and in the presence of $1 \mathrm{mM}$ glucose. There is no obvious reaction peak current observed for the GO modified GCEs either in the absence or presence of glucose. However, a pair of well-defined redox peaks with a cathodic peak at $361 \mathrm{mV}$ and the corresponding anodic peak at 441 
$\mathrm{mV}$ is observed at the Ni-rGO hybrid-modified GCE in the absence of glucose, which are assigned to the $\mathrm{Ni}(\mathrm{III}) / \mathrm{Ni}(\mathrm{II})$ redox couple [12,33].

Figure 3. (a) Low-magnification and (b) high-magnification SEM image of the as-prepared Ni-rGO nanohybrids; (c) Low-magnification and (d) high-magnification TEM image of the as-prepared Ni-rGO nanohybrids.

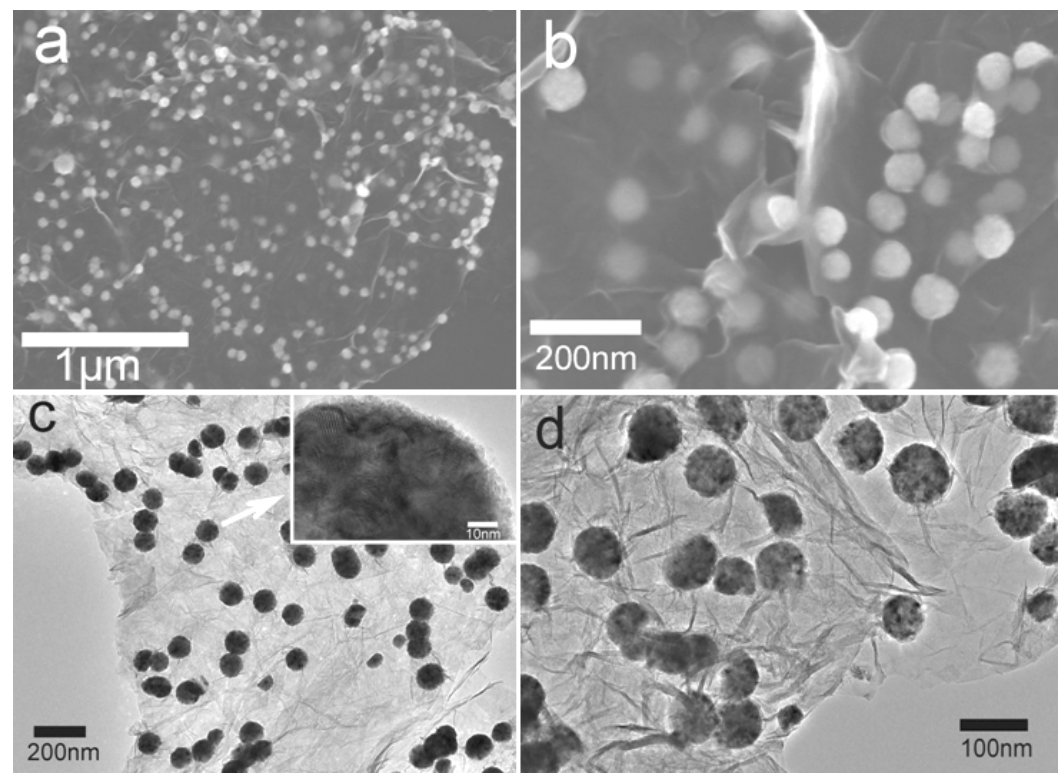

Figure 4. Forty consecutive cyclic voltammograms curves of the N-rGO nanohybridmodified electrode in $0.1 \mathrm{M} \mathrm{NaOH}$ solution at a scan rate of $0.1 \mathrm{Vs}^{-1}$.

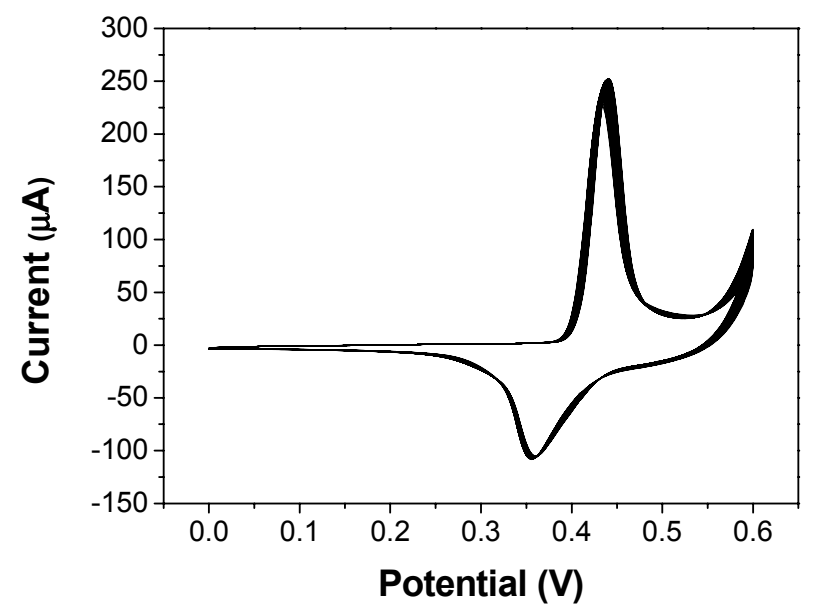

The Ni (II) species on the electrode are due to the oxidation of $\mathrm{Ni}(0)$. Upon addition of $1 \mathrm{mM}$ glucose, there is a great enhancement of the anodic peak current and considerable decrease of cathodic peak current, which indicates that the Ni-rGO hybrids could catalyze the oxidation of glucose. The oxidation of glucose to glucolactone is catalyzed by the $\mathrm{Ni}(\mathrm{III}) / \mathrm{Ni}(\mathrm{II})$ redox couple, where the $\mathrm{Ni}$ (III) species on the electrode surface rapidly oxidizes glucose at the anode, converting $\mathrm{Ni}$ (II) into $\mathrm{Ni}$ (III) species. Thus, the change in concentrations of $\mathrm{Ni}$ (II) and $\mathrm{Ni}$ (III) ratio causes the increase of the anodic peak current and the decrease of the cathodic peak current [11]. In addition, the stability for the Ni-rGO hybrid-modified GCE after activation is also examined by recording 40 consecutive CV curves between 0 and $600 \mathrm{mV}$ in $0.1 \mathrm{M} \mathrm{NaOH}$ at a scan rate of $0.1 \mathrm{Vs}^{-1}$ (Figure 4). No obvious peak 
current change is observed, which demonstrates the Ni-rGO hybrid- modified GCE is very stable.

Figure 5(b) shows the well-defined steady-state amperometric response of the as-prepared Ni-rGO hybrid-modified GCE nonenzymatic glucose sensing with the successive addition of glucose to $0.1 \mathrm{M}$ $\mathrm{NaOH}$ at an applied potential of $0.5 \mathrm{~V}$.

Figure 5. (a) CVs of the as-prepared GO and Ni-rGO nanohybrids modified GCE recorded in $0.1 \mathrm{M} \mathrm{NaOH}$ solution and in $0.1 \mathrm{M} \mathrm{NaOH}$ solution and in the presence of $1 \mathrm{mM}$ glucose (Scan rate: $0.1 \mathrm{Vs}^{-1}$ ), and the amperometric response of the Ni-rGO nanohybrid-modified GCE to successive addition of glucose from: (b) 1 to $110 \mu \mathrm{M}$, and (c) 1 to $10 \mu \mathrm{M}$ into $0.1 \mathrm{M} \mathrm{NaOH}$ with stirring at an applied potential of $0.5 \mathrm{~V}$. The linear relationship between the catalytic current and glucose concentration were inset, respectively. (d) Successive injection of $1 \mathrm{mM}$ glucose and interfering species $0.1 \mathrm{mM} \mathrm{AA}$ and $0.02 \mathrm{mM}$ UA at $100 \mathrm{~s}$ intervals into $0.1 \mathrm{M} \mathrm{NaOH}$ at an applied potential of $0.5 \mathrm{~V}$.
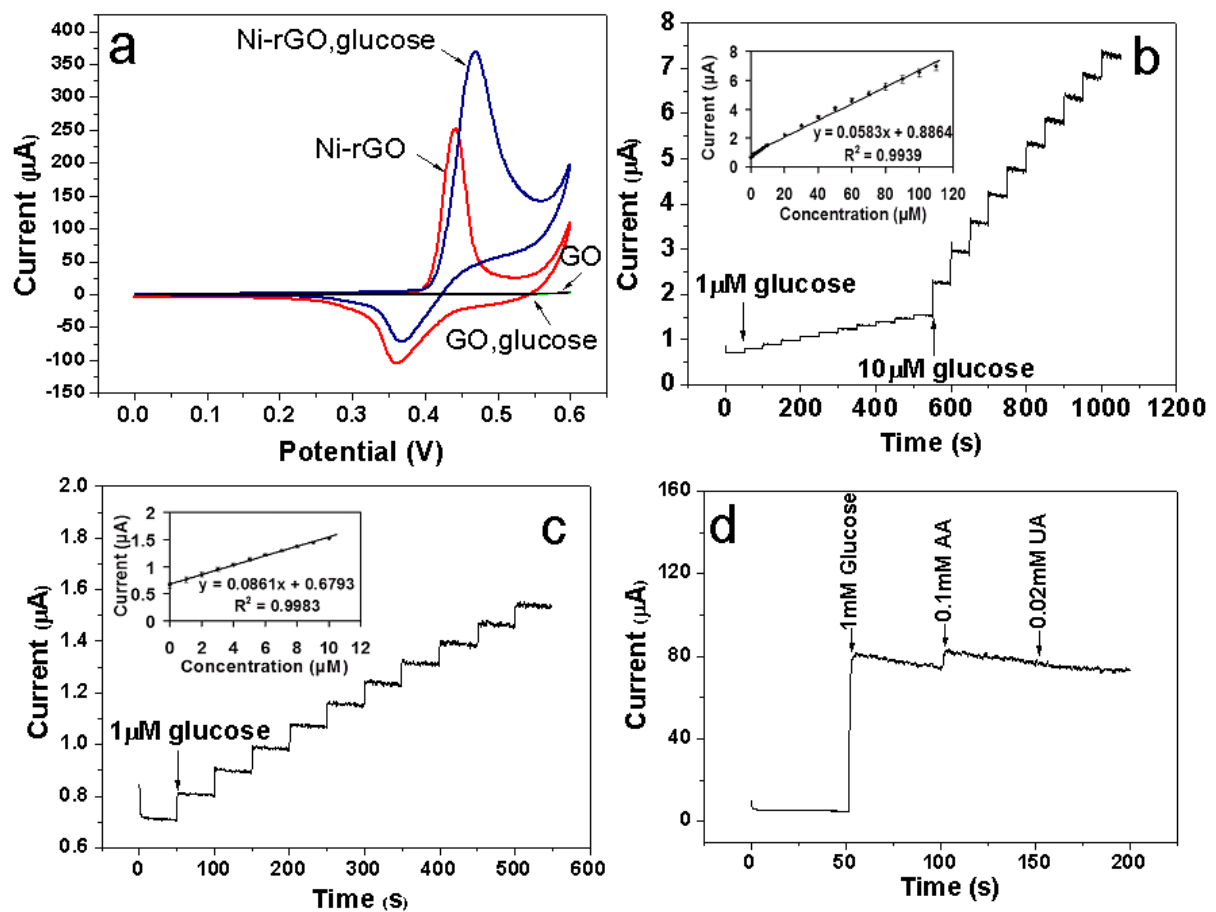

The response time is less than $5 \mathrm{~s}$, revealing a rapid and sensitive response to glucose, which is ascribed to the excellent electrocatalytic properties and rapid charge transfer of the Ni-rGO hybrid electrodes. The calibration plot (inset in Figure 5(b)) has a wide linear range between 1 and $110 \mu \mathrm{M}$ with a gradient of $57.5 \mathrm{nA} \cdot \mu \mathrm{M}^{-1}$ and a correlation coefficient of 0.9939 (the sensitivity is about $813 \mu \mathrm{A} \cdot \mathrm{mM}^{-1} \cdot \mathrm{cm}^{-2}$ ). More detailed examination of Figure $5(\mathrm{c})$ reveals the linear relationship with a better correlation coefficient of 0.9983 (inset in Figure 5(c)) over the lower concentration range of $1-10 \mu \mathrm{M}$. In this case, there is a steeper gradient of $66.2 \mathrm{nA} \cdot \mu \mathrm{M}^{-1}$ corresponding to a higher sensitivity of about $937 \mu \mathrm{A} \cdot \mathrm{mM}^{-1} \cdot \mathrm{cm}^{-2}$. The oxidation of glucose on the Ni surface can be regarded as a direct electrooxidation process. The coverage of $\mathrm{Ni}$ (III) on the $\mathrm{Ni}$ surface increases with the increase of potential. There are enough glucose molecules to adsorb on the Ni(III) sites initially, and which is an adsorption-controlled process that is not sensitive to the concentration. When the potential is high enough, the glucose diffusing to the electrode surface cannot inhabit the active Ni(III) sites completely, 
and which is a diffusion controlled process that is sensitive to the concentration. There is a potential at which the glucose molecules diffusing to the electrode are equal to the amount of Ni(III) sites. It is obviously that a higher potential is needed to form more Ni(III) sites for a higher concentration of glucose. The anodic peak current then shifts positively as the glucose concentration increases [34]. Thus, the Ni-rGO hybrid-modified GCE possesses better sensitivity for lower concentrations of glucose.

Furthermore, it is well known that AA and UA present in the biological samples could be easily oxidized at positive potential and often interfere with the detection of glucose by other methods [35]. To evaluate the selectivity of this sensor, the amperometric response of the Ni-rGO hybrid-modified electrode to UA $(0.02 \mathrm{mM}), \mathrm{AA}(0.1 \mathrm{mM})$, and glucose $(1 \mathrm{mM})$ on the physiological level at an applied potential of $0.5 \mathrm{~V}$ in $0.1 \mathrm{M} \mathrm{NaOH}$ solution is shown in Figure 5d. Compared with glucose, the current responses of these interfering species are very weak and can be neglected. Therefore, the as-prepared Ni-rGO hybrid-modified GCE also exhibits excellent selectivity for glucose detection.

\section{Conclusions}

In summary, we have presented a new and facile method to prepare hybrid nanostructures of $\mathrm{Ni}$ nanospheres deposited onto rGO sheets (Ni-rGO), by combining Ni precipitation and GO reduction in one single step via a rapid microwave-assisted process. The Ni nanospheres are uniform in size and well-dispersed onto the rGO surfaces. The GCE electrodes modified using these Ni-rGO hybrids exhibited high sensitivity, fast response and excellent stability for nonenzymatic determination of glucose. The simple preparation procedures, low cost and enhanced electrocatalytic activity can potentially pave the way for inexpensive, effective and highly sensitive glucose sensors.

\section{Acknowledgments}

Financial supports from the Natural Science Foundation of China (21171146) and Zhejiang Provincial Natural Science Foundation of China (Y4110304). Thanks are also due to National Research Foundation, Singapore under the grant NRF-CRP-G-2007-01.

\section{References}

1. Turner, A.P. F.; Chen, B.N.; Piletsky, S.A. In vitro diagnostics in diabetes: meeting the challenge. Clin. Chem. 1999, 45, 1596-1601.

2. Richter, E.R. Biosensors: Applications for dairy food industry. J. Dairy Sci. 1993, 76, 3114-3117.

3. Liu, Y.; Wang, M.K.; Zhao, F.; Xu, Z.A.; Dong, S.J. The direct electron transfer of glucose oxidase and glucose biosensor based on carbon nanotubes/chitosan matrix. Biosens. Bioelectron. 2005, 21, 984-988.

4. You, C.P.; Xu, X.; Tian, B.Z.; Kong, J.L.; Zhao. D.Y.; Liu, B.H. Electrochemistry and biosensing of glucose oxidase based on mesoporous carbons with different spatially ordered dimensions. Talanta 2009, 78, 705-710.

5. Si, P.; Ding, S.J.; Yuan, J.; Lou, X.W.; Kim, D.H. Hierarchically structured one-dimensional $\mathrm{TiO}_{2}$ for protein immobilization, direct electrochemistry, and mediator-free glucose sensing. ACS Nano 2011, 5, 7617-7626. 
6. Wilson, R.; Turner, A.P.F. Glucose oxidase: an ideal enzyme. Biosens. Bioelectron. 1992, 7, 165-185.

7. Jiang, L.C.; Zhang, W.D. A highly sensitive nonenzymatic glucose sensor based on $\mathrm{CuO}$ nanoparticles-modified carbon nanotube electrode. Biosens. Bioelectron. 2010, 25, 1402-1407.

8. Chen, J.; Zhang, W.D.; Ye, J.S. Nonenzymatic electrochemical glucose sensor based on $\mathrm{MnO}_{2} / \mathrm{MWNTs}$ nanocomposite. Electrochem. Commun. 2008, 10, 1268-1271.

9. Xu, F.G.; Cui, K.; Sun, Y.J.; Guo, C.L.; Liu, Z.L.; Zhang, Y.; Shi, Y.; Li, Z. Facile synthesis of urchin-like gold submicrostructures for nonenzymatic glucose sensing. Talanta 2010, 82, 1845-1852.

10. Guo, C.F.; Hu, Y.; Liu, Y.; Mu, Y.; Miao, Y.; Hu, X. Synthesis of MWCNT/nickel glycolate polymer core-shell nanostructures and their nonenzymatic electrocatalytic activity toward glucose. Mater. Chem. Phys. 2011, 130, 10-13.

11. Lu, L.M.; Zhang, L.; Qu, F.L.; Lu, H.X.; Zhang, X.B.; Wu, Z.S.; Huan, S.Y.; Wang, Q.A.; Shen, G.L.; Yu, R.Q. A nano-Ni based ultrasensitive nonenzymatic electrochemical sensor for glucose: Enhancing sensitivity through a nanowire array strategy. Biosens. Bioelectron. 2009, 25, 218-223.

12. Mu, Y.; Jia, D.L.; He, Y.Y.; Miao, Y.Q.; Wu, H.L. Nano nickel oxide modified non-enzymatic glucose sensors with enhanced sensitivity through an electrochemical process strategy at high potential. Biosens. Bioelectron. 2011, 26, 2948-2952.

13. Hutton, L.A.; Vidotti, M.; Patel, A.N.; Newton, M.E.; Unwin, P.R.; Macpherson, J.V. Electrodeposition of nickel hydroxide nanoparticles on boron-doped diamond electrodes for oxidative electrocatalysis. J. Phys. Chem. C. 2011, 115, 1649-1658.

14. Salimi, A.; Roushani, M. Non-enzymatic glucose detection free of ascorbic acid interference using nickel powder and nafion sol-gel dispersed renewable carbon ceramic electrode. Electrochem. Commun. 2005, 7, 879-887.

15. Geim, A.K.; Novoselov, K.S. The rise of graphene. Nature Mater. 2007, 6, 183-191.

16. Park, S.J.; Ruoff, R.S. Chemical methods for the production of graphenes. Nature Nanotech. 2009, 4, 217-224.

17. Shan, C.S.; Yang, H.F.; Han, D.X.; Zhang, Q.X.; Ivaska, A.; Niu, L. Graphene/AuNPs/chitosan nanocomposites film for glucose biosensing. Biosens. Bioelectron. 2010, 25, 1070-1074.

18. Gao, H.C.; Xiao, F.; Ching, C.B.; Duan, H.W. One-step electrochemical synthesis of PtNi nanoparticle-graphene nanocomposites for nonenzymatic amperometric glucose detection. ACS Appl. Mater. Interfaces 2011, 3, 3049-3057.

19. Yang, H.B.; Guai, G.H.; Guo, C.X.; Song, Q.L.; Jiang, S.P.; Wang, Y.L.; Zhang, W.; Li, C.M. $\mathrm{NiO} /$ graphene composite for enhanced charge separation and collection in p-type dye sensitized solar cell. J. Phys. Chem. C 2011, 115, 12209-12215.

20. Lu, Q.; Dong, X.C.; Li, L.J.; Hu, X. Direct electrochemistry-based hydrogen peroxide biosensor formed from single-layer graphene nanoplatelet-enzyme composite film. Talanta 2010, 82, 1344-1348.

21. Zhang, Y.; Gomez, L.; Ishikawa, F.N.; Madaria, A.; Ryu, K.; Wang, C.; Badmaev, A.; Zhou, C.W. Comparison of graphene growth on single-crystalline and polycrystalline $\mathrm{Ni}$ by chemical vapor deposition. J. Phys. Chem. Lett. 2010, 1, 3101-3107. 
22. Lu, C.C.; Jin, C.; Lin, Y.C.; Huang, C.R.; Suenaga, K.; Chiu, P.W. Characterization of graphene grown on bulk and thin film nickel. Langmuir 2011, 27, 13748-13753.

23. Hu, Y.; Liu, Y.; Qian, H.S.; Li, Z.Q.; Chen, J.F. Coating colloidal carbon spheres with CdS nanoparticles: Microwave-assisted synthesis and enhanced photocatalytic activity. Langmuir 2010, 26, 18570-18575.

24. Liu, Y.; Zhou, L.; Hu, Y.; Guo, C.F.; Qian, H.S.; Zhang, F.M.; Lou, X.W. Magnetic-field induced formation of $1 \mathrm{D} \mathrm{Fe}_{3} \mathrm{O}_{4} / \mathrm{C} / \mathrm{CdS}$ coaxial nanochains as highly efficient and reusable photocatalysts for water treatment. J. Mater. Chem. 2011, 21, 18359-18364.

25. Baghbanzadeh, M.; Carbone, L.; Cozzoli, P. D.; Kappe, C. O. Microwave-assisted synthesis of colloidal inorganic nanocrystals. Angew. Chem. Int. Ed. 2011, 50, 11312-11359.

26. Zhao, B.; Song, J. S.; Liu, P.; Xu, W. W.; Fang, T.; Jiao, Z.; Zhang H. J.; Jiang, Y. Monolayer graphene/NiO nanosheets with two-dimension structure for supercapacitors. J. Mater. Chem. 2011, 21, 18792-18798.

27. Hummers, W.S.; Offeman, R.E. Preparation of graphitic oxide. J. Am. Chem. Soc. 1958, 80, 1339-1339.

28. Xu, Y.X.; Zhao, L.; Bai, H.; Hong, W.J.; Li, C.; Shi, G.Q. Chemically converted graphene induced molecular flattening of 5,10,15,20-Tetrakis(1-methyl-4-pyridinio) porphyrin and its application for optical detection of cadmium(II) ions. J. Am. Chem. Soc. 2009, 131, 13490-13497.

29. Park, S.J.; An, J.H.; Jung, I.H.; Piner, R.D.; An, S.J.; Li, X.S.; Velamakanni, A.; Ruoff, R.S. Colloidal suspensions of highly reduced graphene oxide in a wide variety of organic solvents. Nano lett. 2009, 9, 1593-1597.

30. Ren, P.G.; Yan, D.X.; Ji, X.; Chen, T.; Li, Z.M. Temperature dependence of graphene oxide reduced by hydrazine hydrate. Nanotechnology. 2011, 22, 055705.

31. Li, B.J.; Cao, H.Q.ZnO@graphene composite with enhanced performance for the removal of dye from water. J. Mater. Chem. 2011, 21, 3346-3349.

32. Chang, K.H.; Lee, Y.F.; Hu, C.C.; Chang, C.I.; Liu, C.L.; Yang, Y.L. A unique strategy for preparing single-phase unitary/binary oxides-graphene composites. Chem. Commun. 2010, 46, 7957-7959.

33. Zhao, C.Z.; Shao, C.L.; Li, M.H.; Jiao, K. Flow-injection analysis of glucose without enzyme based on electrocatalytic oxidation of glucose at a nickel electrode. Talanta 2007, 71, 1769-1773.

34. Wang, C.X.; Yin, L.W.; Zhang, L.Y.; Gao, R. Ti/TiO 2 Nanotube array/Ni composite electrodes for nonenzymatic amperometric glucose sensing. J. Phys. Chem. C 2010, 114, 4408-4413.

35. Chen, X.M.; Lin, Z.J.; Chen, D.J.; Jia, T.T.; Cai, Z.M.; Wang, X.R.; Chen, X.; Chen, G.N.; Oyama, M. Nonenzymatic amperometric sensing of glucose by using palladium nanoparticles supported on functional carbon nanotubes. Biosens. Bioelectron. 2010, 25, 1803-1808.

(C) 2012 by the authors; licensee MDPI, Basel, Switzerland. This article is an open access article distributed under the terms and conditions of the Creative Commons Attribution license (http://creativecommons.org/licenses/by/3.0/). 